\section{Cumulus clouds and UV-B}

SIR - - It is well known that clouds can attenuate solar ultraviolet-B (UV-B) radiation at the surface ${ }^{1}$. Less well known is that scattering from the sides of cumulus clouds can enhance total (global) solar irradiance by $20 \%$ or more over the maximum solar noon value ${ }^{2}$. This phenomenon occurs in the UV-B ${ }^{3}$, and here we report brief (about 0.5-hour), biologically significant increases.

During the 1994 Hawaii Ultraviolet Survey, the first in a planned annual series of observations, 10 miniature UV-B $(310 \mathrm{~nm})$ total-sky radiometers were placed in lava at elevations ranging from sea level to $3.4 \mathrm{~km}$ across the island of Hawaii from 11 to 24 June 1994. Two pairs of 940 - and $1,(020-\mathrm{nm}$ total sky radiometers were used to detect the presence of total water vapour and cirrus clouds during the entire study, one pair at the Mauna Loa Observatory (elevation $3.4 \mathrm{~km}$ ) and days. cloud-free day. The integrated increase in UV-B caused by cumulus clouds was compensated for by blockage of the solar disk by clouds. Cumulus clouds arrived over the observatory at 12:01 local time, and from then until 16:01 local time the mean total-sky UV-B was only $-0.7 \%$ below that on the cumulus-free day.

The significance of these observations is that the two episodes of increased UV-B occurred at or near solar noon and their duration was sufficient to reduce the time required for erythaema (reddening) of exposed human skin by at least $15 \%$ below that expected under an identical ozone column and a clear sky. Similar enhancements of UV-B caused by cumulus clouds were observed in August 1994 at Seguin in south-central Texas, but only on haze-free

One approach to treating the effects of clouds is to estimate the attenuation pro-

Total-sky UV-B radiation at 310 $\mathrm{nm}$ at Mauna Loa Observatory on 19 and 22 June 1994 (smooth and jagged lines, respectively). Increases in UV-B on 22 June are caused by reflection from cumulus clouds. Decreases are caused by clouds blocking the solar disk. Observations are by 1 of 10 miniature $(1.7 \times 5 \times 5 \mathrm{~cm})$ UV-B radiometers developed for this survey. Each instrument can accept interchangeable, water-resistant, plug-in irradiance probes (1.9 dia-

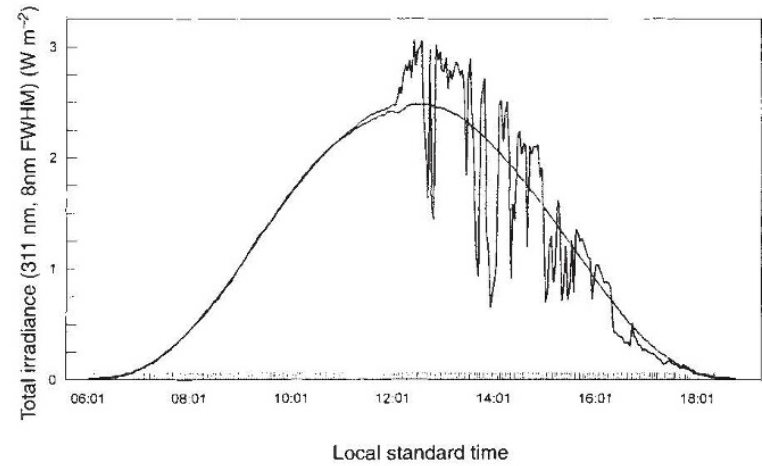

meter $\times 4 \mathrm{~cm})$. UV-B is detected by a narrow bandpass $(7-\mathrm{nm})$ interference filter with less than $2 \%$ UV-A leakage. A near-cosine response is provided by a snap-on plastic (Teflon) diffuser. The photodiode amplifier design is similar to one described elsewhere ${ }^{4}$. Each instrument includes a programmable microcomputer (Onset Computer Corp.) capable of storing 1,800 samples of peak or average irradiance during intervals ranging from $0.5 \mathrm{~s}$ to several hours. During the 1994 Hawaii UV-B Survey, peak and average observations of UV-B at 2-s intervals were also made at various depths under water. These observations and the design of the new radiometer will be described elsewhere.

the other at various sites near sea level.

On several days and at various locations, cumulus clouds increased solar UV$\mathrm{B}$ by $25 \%$ or more over the expected value. The figure shows the total-sky UV-B at Mauna Loa Observatory on a day free of cumulus clouds and a subsequent day when cumulus clouds appeared shortly before solar noon (12:22 local time) and persisted through the afternoon. Dips in irradiance occurred when a cloud blocked the solar disk. Significant increases in irradiance occurred when cumulus clouds were near the Sun. The mean increase over the expected irradiance from $12: 13$ to $12: 34$ local time was $16.8 \%$ (peak, $23.6 \%$ ). The mean increase over the expected irradiance from 12:51 to $13: 25$ was $16.5 \%$ (peak, $22.8 \%$ ). The highest cloud-induced increase during midday occurred at $14: 51$ when total UVB was $29.8 \%$ above that on the previous vided by a cloudy sky using regression erties such as fractional cloud cover. This method is most similar to that used to produce the US ultraviolet index made available to the public on a daily basis since 28 June 1994. A deficiency of this method is that it cannot produce an irradiance in excess of the value for clear skies.

Enhancement of UV-B by cumulus clouds is not necessarily evident in measurements made by commonly used time-integrating UV-B radiometers and especially in predictions based solely on satellite ozone measurements. Therefore,

\footnotetext{
1. Frederick, J. E. \& Snell, H. E. J. Clim. 3, 373-381 (1990)

2. Segal, M. \& Davis, J. J. appl. Meteor. 31, 217-222 (1992)

3. Nack, M. L. \& Green, A. E. S. Appl. Opt. 13, 2405-2415 (1974).
}

4. Mims, F. M. Scient. Am. 263(2), 106-109 (1990) models based on readily observable prop- the general public should be advised that cumulus clouds near the Sun can significantly intensify solar UV-B, particularly during summer and especially when both the Sun and cumulus clouds are near the zenith.

\section{Forrest M. Mims III}

Sun Photometer Atmospheric Network, Seguin, Texas 78155, USA

John E. Frederick

Department of Geophysical Sciences,

University of Chicago,

Chicago, Illinois 60637, USA

\section{Calcium signals in neurons}

SIR - Al-Mohanna et al. ${ }^{1}$ state that previous reports ${ }^{2-5}$ of nuclear amplification of $\mathrm{Ca}^{2+}$ in neurons are due to measurement artefact, and claim that nuclear $\mathrm{Ca}^{2+}$ signals are actually lower than cytoplasmic $\mathrm{Ca}^{2+}$ signals upon depolarization. Here we discuss the important question of whether or not nuclear $\mathrm{Ca}^{2+}$ might be autonomous by examining a technical problem which could undermine this conclusion $^{1}$

A key technical consideration is whether disruption of the cell membrane with a patch pipette or a sharp microelectrode can damage the cells under investigation and so cause an increase in $\mathrm{Ca}^{2+}$ over the normal baseline. The data of Al-Mohanna et al. indeed show an important effect of $\mathrm{Ca}^{2+}$ - indicator dye sequestration, but because the physiological status of their N1E-115 neuroblastoma cells and adult rat dorsal root ganglion (DRG) neurons was not evaluated, it is possible that the cells were either injured or stimulated, or had their $\mathrm{Ca}^{2+}$ signals activated as a consequence of micropipette loading of dye.

To support their data, Al-Mohanna et al. present a simple model of centripetal $\mathrm{Ca}^{2}+$ diffusion which does not take into account $\mathrm{Ca}^{2+}$-induced $\mathrm{Ca}^{2+}$ release (CICR), the activity of $\mathrm{Ca}^{2+}$-binding proteins, and the limited diffusion of $\mathrm{Ca}^{2+}$ in cytosol $^{6}$. It is possible that CICR may have been activated in their neurons; an initial small influx of $\mathrm{Ca}^{2+}$ can be substantially amplified by CICR in neurons ${ }^{3,7}$, including adult rat DRG neurons ${ }^{8}$. These elevated $\mathrm{Ca}^{2+}$ signals can last for tens of minutes ${ }^{9}$, offsetting baseline $\mathrm{Ca}^{2+}$ signals at rest.

We tested the possibility that micropipette disruption of the cell membrane might alter baseline levels of $\mathrm{Ca}^{2+}$ by non-disruptively loading adult rat DRG neurons with Fluo-3AM and confocally imaging the neurons before and after sharp microelectrode penetration ( $n=$ 12 ). On disrupting the cell membrane with the micropipette, overall $\mathrm{Ca}^{2+}$ signals increased by $103 \%$. These results suggest 